\title{
The influence of category coherence on inference about cross-classified entities
}

\author{
Andrea L. Patalano, Steven M. Wengrovitz, and Kirsten M. Sharpes \\ Wesleyan University, Middletown, Connecticut
}

\begin{abstract}
A critical function of categories is their use in property inference (Heit, 2000). However, one challenge to using categories in inference is that most entities in the world belong to multiple categories (e.g., Fido could be a dog, a pet, a mammal, or a security system). Building on Patalano, Chin-Parker, and Ross (2006), we tested the hypothesis that category coherence (the extent to which category features go together in light of prior knowledge) influences the selection of categories for use in property inference about cross-classified entities. In two experiments, we directly contrasted coherent and incoherent categories, both of which included cross-classified entities as members, and we found that the coherent categories were used more readily as the source of both property transfer and property extension. We conclude that category coherence, which has been found to be a potent influence on strength of inference for singly classified entities (Rehder \& Hastie, 2004), is also central to category use in reasoning about novel cross-classified ones.
\end{abstract}

A critical function of categories is their use in property inference (Heit, 2000; L. Ross \& Nisbett, 1991; E. E. Smith \& Medin, 1981). This occurs in two ways. First, most straightforwardly, because categories are associated with known properties, when a new entity is encountered and classified as a member of a category, properties of that category can be transferred readily to the entity. For instance, when a neighborhood dog is determined to be a golden retriever, existing knowledge of golden retrievers can be transferred to the dog, including what it eats, how much exercise it needs, and how friendly it is. Second, because categories are associated with many known members, when a new property of one or more known members is discovered, it can be extended readily to other members and to the category as a whole. For instance, learning that a friend's golden retriever, Fido, has a hip disorder might lead one to believe that this problem both explains another golden retriever's health problems and is common among golden retrievers. Categories critically support both the transfer of known properties to new members, which we refer to as property transfer, and the extension of new properties across known members, which we refer to as property extension. Tasks involving property transfer and property extension have been the basis of much research.

Although inference research has focused largely on entities belonging to single categories, everyday property inference is made challenging by the fact that most entities belong to multiple categories (Murphy \& Ross, 1999; B. H. Ross \& Murphy, 1999). As a golden retriever, Fido is also a dog and a mammal, and might be a financial asset, a security system, or a jogging buddy, too. In property transfer (i.e., the transfer of a known property to a new category member), what happens when the entity belongs to multiple categories that make different predictions about the entity's properties? An estimate of the likelihood that Fido is well insured will no doubt be much higher if one focuses on financial assets than if one focuses on jogging buddies. And, in property extension (i.e., the extension of a new category property across members), what happens when the property is possessed by an entity that belongs to multiple categories? How does one assess the extent to which it should be transferred to other members of each relevant category? For example, Fido's hip problems might better extend to other golden retrievers, but his excessive thirst might better extend to jogging companions. A significant part of understanding the use of categories in property transfer and extension in everyday contexts consists of explaining how and why some categories are selected for use over others (Heit \& Rubinstein, 1994; Murphy \& Ross, 1999; B. H. Ross \& Murphy, 1999). The broad purpose of the present research is to identify factors that influence category preference in inference in cross-classification contexts.

Past cross-classification research has focused largely on three contextual influences on category selection for use in property inference: property relevance, category accessibility, and category distinctiveness. First, inferences are made more often from the category with most relevance to the property in question (Heit \& Rubinstein, 1994; Kalish \& Gelman, 1992; Murphy \& Ross, 1999; Rehder, 2006; B. H. Ross \& Murphy, 1999). For example, taxonomic categories have been shown to inform inferences about biological properties of animals, whereas ecological ones inform those about behavior (Heit \& Rubinstein, 1994). Second, preference is given to categories with increased 
mental activation (Macrae, Bodenhausen, \& Milne, 1995; Sinclair \& Kunda, 1999; E. R. Smith, Fazio, \& Cejka, 1996), regardless of whether it is due to chronic accessibility or to contextual priming. Third, emphasis for inference is placed on categories that are distinctive (Nelson \& Klutas, 2000; Nelson \& Miller, 1995; van Rijswijk \& Ellemers, 2002), the last of which refers to the relative number of members that each category possesses. This body of work provides evidence that context is important, but it leaves open whether the structure of categories might also play a role in property transfer and extension.

This question is motivated by the fact that research on learning and inference with singly classified entities focuses on category structure, especially the role of category coherence in facilitating preferential classification and strong property inference. According to a prior family resemblance view of category structure, categories are correlated clusters of features (Rosch \& Mervis, 1975), and strength of property inference is a function of the similarity of an entity's features to a category (Osherson, Smith, Wilkie, López, \& Shafir, 1990). A more recent theory-based view emphasizes category coherence, the degree to which features go together in light of prior theoretical, causal, and teleological knowledge (Medin, 1989; Murphy \& Medin, 1985) beyond the role of simple co-occurrence. For example, "lives in water, eats fish, has many offspring, and is small" describes a more coherent category than does "lives in water, eats wheat, has a flat end, and is used for stabbing bugs" (Murphy \& Wisniewski, 1989), because people can more easily explain how the former properties go together. Coherent category structures are learned more quickly and accurately than less coherent ones, above and beyond effects of family resemblance (see Murphy, 2002, for a review), and the role of coherence in category representation has proved to be a rich source of recent theory development.

With singly classified entities, category coherence has been found to support property inference. Rehder and Hastie (2004; see also Rehder, 2006; Rehder \& Burnett, 2005; but see Lassaline, 1996) conducted a study of the extension of new properties across category members. A novel category was presented as a series of properties, and coherence was manipulated through the presence or absence of a description of property relatedness. Assuming that the member itself was also coherent, when a category member with a new property was shown, the property was extended to more other members for coherent relative to noncoherent categories. Using natural categories, Patalano and Ross (2007) conducted a study that instead used natural job and hobby categories that had been rated on coherence by pretest participants (e.g., nurse and comedian were rated high and low in coherence, respectively). The researchers manipulated the number of members of a category that were said to possess a particular new property. They found that, for the high- relative to low-coherence categories, participants required fewer new members possessing the property before they were willing to extend the property to other members. These findings address a key theoretical and practical issue - namely, that of identifying the structural aspects of categories that support strong property inference (Wisniewski, 2002).
We explore here whether the influence of coherence seen with singly classified entities also emerges in property transfer and property extension with cross-classified entities. In other words, in property transfer, are the properties that are transferred to cross-classified entities more likely to come from higher rather than lower coherence categories? Similarly, in property extension, are the properties that come from cross-classified entities more likely to be extended to other members of higher rather than lower coherence categories? More generally, are people more inclined to make inferences involving the most coherent membership categories of cross-classified entities? On the one hand, it seems natural that this strategy would be extended to the more complex context. For a crossclassified entity, coherence might influence assessment of the strength of inference for each category independently, and then the focus of inference could be directed to the stronger category. On the other hand, the use of such a strategy would seem to require activating multiple category representations simultaneously while maintaining separately the entity's properties associated with each category. This strategy might not be cognitively efficient or even feasible when multiple categories are available as potential sources of inference. As a result, individuals might rely instead on contextual strategies that quickly focus the individual on a single category, such as when the property in question serves as a retrieval cue to a category associated with that property or when a chronically accessible category habitually comes to or stays in mind. In determining which one or more should serve as the source of inference, in the absence of any differentiating contextual information, categories might be weighted equally, or one might be chosen arbitrarily.

Given the many strategies likely to be employed in cross-classification situations, the purpose of the present research was not to argue that coherence alone influences category use in inference, but to assess whether coherence is used at all in cross-classification situations. Finding evidence of such an influence would be valuable in that it would lend further support to the claim that coherence underlies people's beliefs about what makes categories informative for inference and thus more likely to be formed in the first place. It would allow us to draw the practically important conclusion that coherence is not simply an ideal about category structure that is activated in the context of evaluating single categories, but that it influences selection of categories for use in complex tasks more typical of everyday reasoning. Although finding an influence of coherence would not diminish the relevance of contextual factors, it would allow researchers to extend beyond contextual influences alone in understanding how individuals reason about cross-classified entities, and it would bring the research more in line with research on singly classified entities. Such a finding could also offer the underpinnings of a process whereby maximally coherent categories develop over time through accumulation of property inferences about initially coherent ones. Any finding of no influence of coherence in a cross-classification context would not undermine the importance of coherence to representations of individual categories and to beliefs 
about their informativeness. It would, however, mean that this factor plays less of a role than is presently assumed in situations that are typical of everyday reasoning, and that single-category effects do not necessarily "scale up" to more complex situations. It would also suggest that factors other than coherence, such as the contextual influences that have been the focus of past research, might instead be the primary sources of influence in reasoning about cross-classified entities.

In the only previous work on coherence and crossclassification, using natural job and hobby categories rated on coherence, Patalano, Chin-Parker, and Ross (2006) paired high- and low-coherence categories with incompatible hypothetical properties: For example, $80 \%$ of soldiers (high coherence) prefer terriers to beagles, whereas $80 \%$ of matchbook collectors (low coherence) prefer beagles to terriers. When asked the most likely preference of an individual in both categories, participants most often selected the property associated with the higher coherence category. Although the results provide evidence that perceived coherence is related to category use in property transfer, conclusions are limited by the fact that coherence ratings are associated with many variables, including homogeneity of members (Haslam, Rothschild, \& Ernst, 2000; Yzerbyt, Corneille, \& Estrada, 2001), category distinctiveness (Nelson \& Miller, 1995), beliefs about category agency and role differentiation among members (Spencer-Rodgers, Hamilton, \& Sherman, 2007), and relational style of members (Lickel, Rutchick, Hamilton, \& Sherman, 2006). Further work is needed to determine a link between a category's coherence structure and its use in property transfer and to broaden the findings to property extension as well. A direct test involving experimenter-created categories in which property relatedness is manipulated would allow an assessment of whether the influence of coherence emerges in property inference situations with cross-classified entities.

In the present work, we conducted two experiments to test the hypothesis that coherence structure influences category use in inference in the context of cross-classified entities. The first focused on property transfer: A novel cross-classified entity was presented whose property could be inferred from either a coherent or an incoherent category of membership; the task was to decide from which category to transfer the property. This task differed from that in the just-described Patalano et al. (2006) study in that it used experimenter-created categories that varied in coherence rather than natural categories rated on coherence.

The second experiment focused on property extension. A property of six cross-classified category members was presented that might extend to other category members as well; the task was to judge the likelihood of property extension to either a coherent or an incoherent membership category. This task was based loosely on single-category property extension tasks, such as that found in Rehder and Hastie (2004), but with cross-classified entities. If coherence influences property inference with cross-classified entities, individuals should show a consistent preference for coherent categories on the forced choice task in Experiment 1 and should judge the likelihood of property extension for coher- ent categories to be higher than that for incoherent ones in Experiment 2. If coherence does not influence property inference, no differences should emerge because coherence was the only manipulated variable; use of any other strategies, such as arbitrary category selection in Experiment 1 or equal weighting of categories in Experiment 2, should produce no systematic coherence preference.

Category materials were the same for both experiments. Activity categories (i.e., jobs and hobbies) were used because people routinely belong to multiple social categories, and thus this was a natural context in which to study cross-classified entities. As in past research, coherent and incoherent categories were matched on number and type of features, and varied only on feature interrelatedness. Specifically, each category was created using a category label (e.g., "a daxetic"), an activity description (e.g., "one who melts glass into jewelry"), one personality-trait feature of category members (e.g., "artistic"), and three behavioral features (though some of the latter were perceptual as well, as in "carries a bow and arrow"). We based the choice of this constellation of features on Dahlgren's (1985) finding that these are the kinds of features typically generated for activity-based categories. Coherence was operationalized as the presence versus absence of a clear relationship between the activity description and each of the four features based on everyday knowledge (similar to Rehder \& Ross, 2001).

\section{EXPERIMENT 1 \\ Property Transfer With Multiple Categories}

The goal of this experiment was to test the hypothesis that coherence influences category selection for use in property transfer when multiple categories are available. The experimental task was to assess which of two categories' mutually exclusive properties was more likely to extend to an entity belonging to both. Specifically, two novel categories, one coherent and one incoherent, were presented. Participants were told about mutually exclusive properties of the two categories, such as that one category's members preferred the color blue to red, whereas the other one's members preferred red to blue. They were asked which of the properties a person belonging to both categories was more likely to have. Although a reasonable strategy in the present situation would be to make a judgment of strength of inference for each category independently and to use the results as a basis for category selection, there were few existing data regarding whether such a strategy would be used. If category coherence influences category preference for inference about cross-classified entities, people should be more likely to extend properties from coherent than from incoherent categories. If coherence does not influence category preference for inference, no differences should emerge, in that other strategies, such as arbitrary selection of a category, should produce no systematic coherence preference.

\section{Method}

Participants. A total of 88 undergraduates (56 female and 32 male; 18-24 years old) at Wesleyan University volunteered in 
exchange for monetary compensation. They worked in groups of 10-20 individuals, in classrooms.

Materials and Procedure. Four problems were created that paired a coherent and an incoherent category (see the Appendix). For half of the participants, the pairs were (coherent category is italicized): penderites-fragglers, migrabeaners-angorists, daxeticsfloritumites, and borinists-ziffers (Set A of materials). For the other half of the participants (Set B of materials), the same pairs were used but the previously incoherent category became the coherent one (as in penderites-fragglers). Incoherent categories were made by combining unrelated behavioral features drawn from multiple coherent categories. Concrete properties that were not obviously associated with the categories were used (as in Patalano et al., 2006). Each property was matched to a single category (e.g., penderites always preferred basketball to baseball), but the category was either coherent or incoherent, depending on the material set. Problems were presented in two counterbalancing orders (see Example 1). A categorical response was collected, and participants were also asked to evaluate their confidence in their response on a 5-point scale where $1=$ not at all confident and $5=$ highly confident. The task took $10 \mathrm{~min}$ to complete and was followed by an unrelated 2-min decision-making task.

\section{Results and Discussion}

No reliable difference in material sets was found $(p>.100)$, so the results were collapsed over material sets. Participants were more likely to choose the coherent categories $(60 \%$ of the time, $S E=3.0)$ than would be expected by chance $[t(87)=4.07, p<.001] ;^{1}$ this pattern held for seven out of the eight different category pairs (with item percentages ranging from $57 \%$ to $72 \%$ ). ${ }^{2}$ Of the 88 participants, 44 selected coherent categories more than half of the time, 31 participants selected coherent categories exactly half the time, and only 13 selected incoherent categories more than half the time. That a moderate number selected coherent categories half of the time is not surprising, given that each participant completed only four problems. There were no differences in confidence ratings for problems in which coherent relative to incoherent categories were selected $(M=3.0, S E=0.18$, for both conditions) $[t(73)=0.50, p=.618]$, with 14 participants excluded for selecting only coherent categories. A reasonable strategy consistent with the data is that individuals, at least implicitly, judge the strength of inference for each category and use the results as a basis for category selection. A strategy that might account for additional variance here and that was mentioned earlier would be simply to have chosen arbitrarily, especially if and when the relative coherence of categories was not obvious. We also raise the

Example 1

Here are two categories and some associated characteristics: DAXETICS

\begin{tabular}{ll}
\hline Artistic & Precise \\
Melt glass into jewelry & Extract ocean minerals \\
Spend time in their garages & Spend time in offices \\
Wear flameproof clothing & Wear nametags \\
Carry recyclable bottles & Carry blood pressure monitors \\
You have just found out that: & \\
$80 \%$ of daxetics prefer daffodils to tulips. \\
$\quad 80 \%$ of floritumites prefer tulips to daffodils. \\
You now meet someone who is both a daxetic and a floritumite. Do you \\
think this person is more likely to prefer daffodils or tulips?
\end{tabular}

possibility that participants sometimes could have been trying to use the contextual strategy of selecting a category whose features seemed most relevant to the new property (as in, e.g., Heit \& Rubinstein, 1994), despite our attempts to limit property relevance. Use of either would have reduced any effect of coherence. Both will be discussed further after Experiment 2.

Experiment 1 considered the role of category coherence and inference in the context of multiple categories with the property transfer task of deciding from which category to transfer a novel property to a cross-classified entity; it was found that the more coherent category was selected the majority of the time. Another frequently used propertyinference task is that of property extension, assessing the strength of transfer of a novel property of a cross-classified entity to other members of each category. This occurs when one is trying to decide, for example, how likely the politically liberal leanings of a few individuals who are both social workers and deer hunters are to extend to other social workers versus to other deer hunters. The purpose of Experiment 2 was to attempt to extend the finding that coherence influences category use in property inference to a situation in which a property must be transferred from some cross-classified individuals to other individuals belonging to only one of the two categories.

\section{EXPERIMENT 2}

\section{Property Extension With Multiple Categories}

The goal of this experiment was to test the hypothesis that stronger property extensions would be made to novel coherent than to incoherent categories in the context of cross-classified entities. Participants read descriptions of pairs of novel categories with one coherent and one incoherent category per pair and were informed that six recently observed individuals who belonged to both categories also shared a novel property. Participants were then asked to assess the likelihood that a new individual belonging to only one of the categories also possessed the same novel property. Half of the problems seen by each participant were coherent problems, in which the new individual belonged only to the coherent category; the other half were incoherent ones in which the new individual belonged only to the incoherent category. As in Experiment 1, one possible strategy was for individuals to assess the strength of inference to the category in question on the basis of coherence. An alternative is that, because people typically might look to contextual factors to first select a category as a source of inference, when no contextual cues were available, they might simply weight the two categories equally. If coherence influences willingness to extend a property to a membership category of cross-classified entities, probability judgments should be higher when the new individual belongs to the coherent category than when he or she belongs to the incoherent one. If coherence does not influence willingness to extend a property, no differences should emerge, in that other strategies should produce no systematic coherence preference; individuals would be expected to give the same judgments for both coherent and incoherent categories. In this experiment, we included informal exit interviews to probe the range of 
Example 2

Here are two categories and some associated characteristics: DAXETICS

\section{Artistic}

Melt glass into jewelry

Spend time in their garages

Wear flameproof clothing

Carry recyclable bottles Coca Cola to Pepsi and half prefer Pepsi to Coca Cola. The first 6 people you encounter who happen to be both daxetics and floritumites prefer Coca Cola to Pepsi. How likely do you think it is that the next daxetic you encounter who is not a floritumite will prefer Coca Cola to Pepsi too?

other reasoning strategies that might be employed. This experiment was similar in method to past property-extension studies (e.g., Rehder \& Hastie, 2004), except for its use of cross-classified rather than singly classified entities.

\section{Method}

Participants. A total of 60 undergraduates (39 female and 21 male; 18-24 years old) at Wesleyan University volunteered in exchange for monetary compensation. They worked in groups of $10-20$ individuals, in classrooms.

Materials and Procedure. The categories and properties used to create the problems were the same as those used in Experiment 1, except that a different pairing of categories to properties was used (see the Appendix). The instructions surrounding the problems differed from those in the previous experiment (see Example 2). Instructions stated that novel properties (e.g., prefers Coca Cola to Pepsi) should be assumed to be equally likely in the population at large. For each problem, participants read the category descriptions, wrote down one other property that they thought might be associated with each category (this was just to make sure they read and processed the categories), and then answered the inference question by writing a probability between $0 \%$ and $100 \%$. Participants completed all problems at their own pace, with one problem per page of a booklet. The task took approximately 15 min to complete and was followed by an unrelated task.

\section{Results and Discussion}

No reliable differences in materials were found ( $p$ s $>.100)$, so the results were collapsed over material sets. Participants generated higher likelihood judgments for the extension of properties to new members of coherent categories $(M=55 \%, S E=2.0)$ than to new members of incoherent ones $(M=47 \%, S E=2.0)[t(59)=3.04$. $p=.003]$; this pattern held for all four category pairs (see Figure 1). Of all 60 participants, 28 had higher mean judgments for coherent categories than for incoherent ones, 20 had the same mean judgments for coherent and incoherent categories (14 said 50\% for all four problems), and 12 had higher mean judgments for incoherent categories. Although the difference in mean judgments was small, the values reflect a qualitatively important difference between concluding that some individual does $(>50 \%)$ versus does not $(<50 \%)$ have a property. The results show that coherence influences one's willingness to extend a property from some entities belonging to multiple categories to an individual belonging to only one of those categories.

Informal exit interviews revealed that another reasoning strategy used by participants was to look for a feature of either the coherent or the incoherent category to which the novel property might be related. For example, if the novel

property was "prefers daffodils to tulips" and being artistic was an existing feature of one of the categories, a perceived link between these aesthetic characteristics might lead one to extend the novel property more liberally for this category. In other words, besides use of overall coherence, a strategy that seemed to have been employed frequently was to try to generate specific explanations for how an existing feature could give rise to a novel property. This strategy plausibly could have been used in Experiment 1 as well. Assigning the same probability to high- and low-coherence categories was also a reasonable behavior, just as choosing arbitrarily was in Experiment 1. Additionally, responses could have been influenced by a task-specific, pragmatic concern that it would seem odd to be told that the first six daxetics and floritumites encountered preferred Coca Cola to Pepsi if this property was, in fact, generalizable only to one of the categories. All of these would have worked against our hypothesis and reduced any coherence effect; they point to what other strategies are used and why the more coherent category is not always selected. The possible use of property relatedness is particularly striking. This contextual strategy might be a particularly powerful and pervasive one, given its emergence even when relatedness information was minimized and coherence differences were magnified.

\section{GENERAL DISCUSSION}

We used two diverse inference tasks, property transfer and property extension, to address the question of whether category coherence influences property inference with

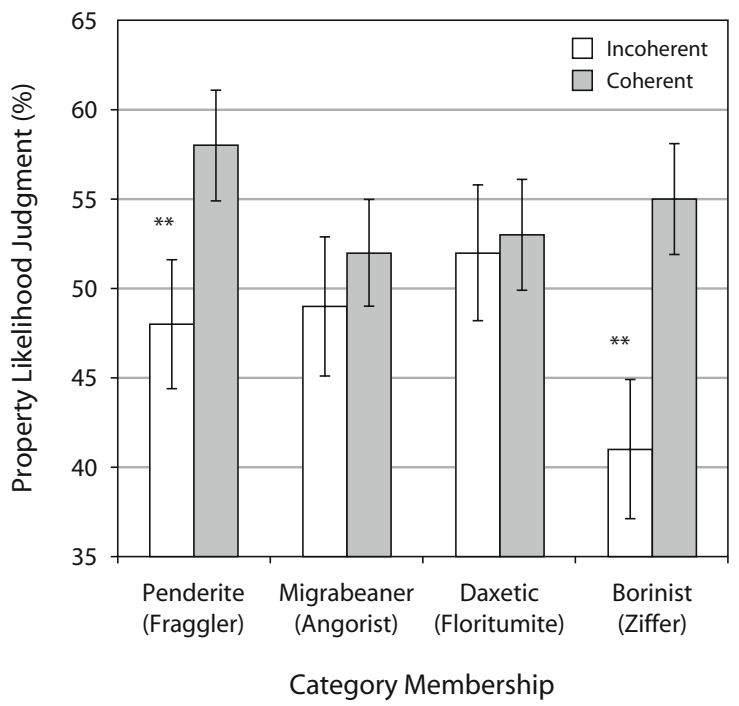

Figure 1. In Experiment 2, mean property likelihood judgment for a new member of an incoherent category in the context of a coherent counterpart (e.g., incoherent penderite and coherent fraggler) versus a new member of a coherent category in the context of an incoherent counterpart (e.g., coherent penderite and incoherent fraggler). Data from participants receiving Set $A$ of the materials contributed to first, fourth, sixth, and seventh bars; data from those with Set $B$ contributed to the remainder. ${ }^{* *} p<.01$ for one-tailed independent-samples $t$ test. 
cross-classified entities. In the first experiment, in which a property could be transferred from either a coherent or an incoherent category, the properties associated with the coherent categories were selected more often. In the second experiment, when properties could be extended to other members of either a coherent or an incoherent category, properties were extended more readily to coherent categories. The present work goes beyond past work with singly classified entities (Patalano \& Ross, 2007; Rehder $\&$ Hastie, 2004) in showing that the influence of coherence on property extension applies to cross-classified entities as well. It also goes beyond the initial cross-classification work of Patalano et al. (2006), who used natural categories and found initial evidence that the coherence of a category influences category selection; the present work rules out possible confounds associated with the use of natural categories. The present findings provide compelling evidence that the general coherence of a category influences property inference for cross-classified entities. The results suggest that the same strategies used in single category situations can be and are used in the context of multiple categories to independently assess the coherence of each category and to give greater weight to the category with stronger influence potential in reasoning. In other words, it is not the case, as we considered earlier, that the presence of multiple categories challenges our ability to make multiple coherence assessments or otherwise extinguishes the use of this strategy.

This finding is important for a number of reasons. First, it adds support to the dominant view that category coherence (i.e., the extent to which category features are interrelated through knowledge) is central to a category's informativeness for inference, a view that has been formed largely through studies of singly classified entities. Second, it supports the novel claim here that coherence is of potential practical relevance in everyday inference situations, which typically involve selecting one from among multiple categories as an inference source. That coherence has been known to influence strength-of-inference judgments about singly classified entities did not ensure that it would also influence category preference for entities in multiple categories, given the many differences between situations and, particularly, the added complexity of the cross-classification situation. Third, the results bolster the argument that coherence plays a role in the development of inferentially rich categories by focusing attention on some categories over others early in category use as preferred sources of property transfer and inference. This argument would be untenable if there were no influence of coherence on inference about cross-classified entities, especially given that children as young as 4 years old have been shown to represent and use cross-classified entities (Nguyen, 2007).

In the two experiments presented here, the magnitude of the difference was modest (e.g., $60 \%$ selected the coherent category in Experiment 1). This does not diminish the fact that coherence was shown to influence behavior here and that, in fact, the magnitude of that influence was not greatly different from that found in past work (e.g., $66 \%$ selected the coherent category in Patalano et al., 2006).
However, the results do remind us that coherence was not likely the only strategy employed. As suggested in Experiment 2, a likely major contributor to the small size of the effect in both studies is that participants were using a second strategy (i.e., trying to assess which category already possessed a property that seemed at least plausibly related to the new property in question). For example, for the new property "a preference for tulips over daffodils," there was a suggestion in the data that it was more readily extended to category members who were artistic than to those who were precise. Use of such a strategy is consistent with past findings indicating that reasoning about cross-classified entities is influenced by property-specific explanations (Heit \& Rubinstein, 1994; B. H. Ross \& Murphy, 1999). For example, B. H. Ross and Murphy found that a category was more likely to be selected for inference about a food item if the type of category (e.g., taxonomic vs. script) supported the type of property about which an inference was to be made (e.g., vitamin content vs. where it might be served). In the present studies, although the properties were intended to be unrelated to the categories, it was difficult to ensure that this was the case in a domain in which people could bring to bear considerable general knowledge. Rehder (2006) recently contrasted factors of similarity (of entity to category) and property relatedness and found that effects of similarity on property extension were largely erased when the new property was related causally to an existing category property. The present results suggest that, like overall similarity, use of coherence might also be a general strategy that is engaged most heavily when more specific contextual information cannot be brought to bear. When specific information is present, its use might sometimes compete with the use of general coherence.

There are two important limitations to this work. The first is that we have tried to remain largely agnostic on the issue of what types of feature interrelations promote property inference most, instead deriving our operationalization of coherence from past work on natural activity categories. In other research, coherence has been found to facilitate learning and use whether the relations that link category features are causal (Ahn, 1998; Rehder \& Hastie, 2001, 2004), spatial or temporal (Lin \& Murphy, 2001), related to an abstract theme (Erickson, Chin-Parker, \& Ross, 2005; Rehder \& Ross, 2001), or based on goals (Barsalou, 1983, 1985). Some have argued that causal features have a special status (Ahn, 1998; Ahn \& Kim, 2000; Ahn, Kim, Lassaline, \& Dennis, 2000; but see Rehder \& Hastie, 2004), and others have found that common, deep underlying features among members increase belief in common surface features and vice versa (e.g., Yzerbyt et al., 2001; Yzerbyt, Rogier, \& Fiske, 1998), following an essentialist perspective (e.g., Medin \& Ortony, 1989; Rothbart \& Taylor, 1992). Although the categories used here could be construed as representing deep underlying personality traits that give rise to surface features, these materials differed from some used in the past (e.g., Yzerbyt et al., 1998) in that the presence of deep and surface features was the same for both coherent and incoherent categories here; it was only the ease of construction of causal links that differed 
across category types. Given this, the results suggest that the coherence effect on category selection in the present study is driven by something other than the presence of either deep or surface features; but, whether this something else is a common-cause structure versus more general feature interrelatedness, we are unable to determine.

Another important limitation of the present experiments is that they addressed incoherent categories but not noncoherent ones. To appreciate this distinction, consider the difference between an animal with wings and a red crest versus one with wings and a tendency to burrow in the ground. The first animal is noncoherent in that it is not clear how wings and a red crest are related, but there is nothing striking about their co-occurrence; whereas the other is incoherent in that wings and burrowing are mutually inconsistent by most people's accounts of the world. If incoherent categories are just plain ill-formed, individuals might be biased toward selecting coherent categories in response to nearly any question asked. Although we raise this as a possibility, we believe it to have been unlikely here, in that all categories were deemed odd (people holding meditative poses in trees was, after all, a coherent category); there are many a priori reasons for selecting an incoherent category, including its greater distinctiveness (e.g., Nelson \& Miller, 1995) and its broader spread of features for use in explaining new properties (e.g., Rehder, 2006), and the same coherent categories were given similar property judgments in unpublished studies involving singly categorizable entities. Nonetheless, this is an important distinction for future consideration, especially because it pertains to whether incoherent categories are part of a coherence continuum (from more to less coherent) versus treated quite differently.

This work contributes to a growing body of research on factors influencing category selection for inference in cross-classification contexts. Past research on this topic has focused largely on the contextual influence of category relevance to the property in question, as well as on those of category accessibility (Macrae et al., 1995; Sinclair \& Kunda, 1999; E. R. Smith et al., 1996) and category distinctiveness (Nelson \& Klutas, 2000; Nelson \& Miller, 1995; van Rijswijk \& Ellemers, 2002). The present work provides evidence that the structure of the category itself, rather than only the context in which it occurs, is also important for understanding when and how a category is selected for inference. In balance, however, the present work also suggests the influence of the category's relevance to the property in question as a potentially major influence on category selection that might often compete with category coherence. An important challenge for future work will be to integrate individual influences into a broader understanding of when and how multiple categories are retrieved from memory, how contextual and structural category factors jointly influence reasoning about available categories, and when and how information from multiple categories is integrated versus the fact that categories are selected individually for use in inference-related judgments. This course of research will allow us to describe more fully and richly the process by which reasoning occurs in cross-classification contexts.

\section{AUTHOR NOTE}

We thank Nancy S. Kim and three anonymous reviewers for very helpful comments on an earlier draft of this article. Correspondence concerning this article should be addressed to A. L. Patalano, Department of Psychology, Wesleyan University, 207 High St., Middletown, CT 06459 (e-mail: apatalano@wesleyan.edu).

\section{REFERENCES}

AHN, W.-K. (1998). Why are different features central for natural kinds and artifacts? The role of causal status in determining feature centrality. Cognition, 69, 135-178.

AHN, W.-K., \& KIM, N. S. (2000). The causal status effect in categorization: An overview. In D. L. Medin (Ed.), The psychology of learning and motivation (Vol. 40, pp. 23-65). San Diego: Academic Press.

Ahn, W.-K., Kim, N. S., Lassaline, M. E., \& Dennis, M. J. (2000). Causal status as a determinant of feature centrality. Cognitive Psychology, 41, 361-416.

Barsalou, L. W. (1983). Ad hoc categories. Memory \& Cognition, 11, 211-227.

BArsalou, L. W. (1985). Ideals, central tendency, and frequency of instantiation as determinants of graded structure in categories. Journal of Experimental Psychology: Learning, Memory, \& Cognition, 11, 629-654.

DAHLGREN, K. (1985). The cognitive structure of social categories. Cognitive Science, 9, 379-398.

Erickson, J. E., Chin-PARker, S., \& Ross, B. H. (2005). Inference and classification learning of abstract coherent categories. Journal of Experimental Psychology: Learning, Memory, \& Cognition, 31, 86-99.

Haslam, N., RothschiLd, L., \& ERnst, D. (2000). Essentialist beliefs about social categories. British Journal of Social Psychology, 39, 113-127.

HeIt, E. (2000). Properties of inductive reasoning. Psychonomic Bulletin \& Review, 7, 569-592.

Heit, E., \& Rubinstein, J. (1994). Similarity and property effects in inductive reasoning. Journal of Experimental Psychology: Learning, Memory, \& Cognition, 20, 411-422.

HogG, R. V., \& CRAIG, A. T. (1995). Introduction to mathematical statistics (5th ed.). Englewood Cliffs, NJ: Prentice-Hall.

Kalish, C. W., \& Gelman, S. A. (1992). On wooden pillows: Multiple classification and children's category-based inductions. Child Development, 63, 1536-1557.

LASSAline, M. E. (1996). Structural alignment in induction and similarity. Journal of Experimental Psychology: Learning, Memory, \& Cognition, 22, 754-770.

Lickel, B., Rutchick, A. M., Hamilton, D. L., \& Sherman, S. J. (2006). Intuitive theories of group types and relational principles. Journal of Experimental Social Psychology, 42, 28-39.

Lin, E. L., \& MurPhy, G. L. (2001). Thematic relations in adults' concepts. Journal of Experimental Psychology: General, 130, 3-28.

Macrae, C. N., Bodenhausen, G. V., \& Milne, A. B. (1995). The dissection of selection in person perception: Inhibitory processes in social stereotyping. Journal of Personality \& Social Psychology, 69, 397-407.

Medin, D. L. (1989). Concepts and conceptual structure. American Psychologist, 44, 1469-1481.

Medin, D. L., \& Ortony, A. (1989). Psychological essentialism. In S. Vosniadou \& A. Ortony (Eds.), Similarity and analogical reasoning (pp. 179-195). New York: Cambridge University Press.

MurPhy, G. L. (2002). The big book of concepts. Cambridge, MA: MIT Press.

Murphy, G. L., \& Medin, D. L. (1985). The role of theories in conceptual coherence. Psychological Review, 92, 289-316.

Murphy, G. L., \& Ross, B. H. (1999). Induction with cross-classified categories. Memory \& Cognition, 27, 1024-1041.

MurPhy, G. L., \& WISNIEWSKI, E. J. (1989). Feature correlations in conceptual representations. In G. Tiberghien (Ed.), Advances in cognitive science: Vol. 2. Theory and applications (pp. 23-45). Chichester, U.K.: Horwood.

Nelson, L. J., \& Klutas, K. (2000). The distinctiveness effect in social interaction: Creation of a self-fulfilling prophecy. Personality \& Social Psychology Bulletin, 26, 126-135. 
Nelson, L. J., \& Miller, D. T. (1995). The distinctiveness effect in social categorization: You are what makes you unusual. Psychological Science, 6, 246-249.

NGuyen, S. P. (2007). Cross-classification and category representation in children's concepts. Developmental Psychology, 43, 719-731.

Osherson, D. N., Smith, E. E., Wilkie, O., López, A., \& Shafir, E. (1990). Category-based induction. Psychological Review, 97, $185-$ 200.

Patalano, A. L., Chin-Parker, S., \& Ross, B. H. (2006). The importance of being coherent: Category coherence, cross-classification, and reasoning. Journal of Memory \& Language, 54, 407-424.

Patalano, A. L., \& Ross, B. H. (2007). The role of category coherence in experience-based prediction. Psychonomic Bulletin \& Review, 14, 629-634.

REHDER, B. (2006). When similarity and causality compete in categorybased property generalization. Memory \& Cognition, 34, 3-16.

Rehder, B., \& Burnett, R. C. (2005). Feature inference and the causal structure of categories. Cognitive Psychology, 50, 264-314.

Rehder, B., \& Hastie, R. (2001). Causal knowledge and categories: The effects of causal beliefs on categorization, induction, and similarity. Journal of Experimental Psychology: General, 130, 323-360.

Rehder, B., \& Hastie, R. (2004). Category coherence and categorybased property induction. Cognition, 91, 113-153.

ReHDER, B., \& Ross, B. H. (2001). Abstract coherent categories. Journal of Experimental Psychology: Learning, Memory, \& Cognition, 27, 1261-1275.

Rosch, E., \& Mervis, C. B. (1975). Family resemblances: Studies in the internal structure of categories. Cognitive Psychology, 7, 573-605.

Ross, B. H., \& Murphy, G. L. (1999). Food for thought: Crossclassification and category organization in a complex real-world domain. Cognitive Psychology, 38, 495-553.

Ross, L., \& NisbetT, R. E. (1991). The person and the situation: Perspectives of social psychology. New York: McGraw-Hill.

Rothbart, M., \& TAYLOR, M. (1992). Category labels and social reality: Do we view social categories as natural kinds? In G. R. Semin \& K. Fiedler (Eds.), Language, interaction, and social cognition (pp. 11-36). Thousand Oaks, CA: Sage.
SinClAIR, L., \& KUNDA, Z. (1999). Reactions to a Black professional: Motivated inhibition and activation of conflicting stereotypes. Journal of Personality \& Social Psychology, 77, 885-904.

Smith, E. E., \& Medin, D. L. (1981). Categories and concepts. Cambridge, MA: Harvard University Press.

Smith, E. R., FAzIO, R. H., \& CEJKA, M. A. (1996). Accessible attitudes influence categorization of multiply categorizable objects. Journal of Personality \& Social Psychology, 71, 888-898.

Spencer-Rodgers, J., Hamilton, D. L., \& Sherman, S. J. (2007). The central role of entitativity in stereotypes of social categories and task groups. Journal of Personality \& Social Psychology, 92, 369-388.

VAN RiJswiJK, W., \& Ellemers, N. (2002). Context effects on the application of stereotype content to multiple categorizable targets. Personality \& Social Psychology Bulletin, 28, 90-101.

WisniewsKi, E. J. (2002). Concepts and categorization. In H. Pashler \& D. [L.] Medin (Eds.), Stevens'Handbook of experimental psychology (3rd ed., Vol. 2: Memory and cognitive processes, pp. 467-531). New York: Wiley.

Yzerbyt, V. [Y.], Corneille, O., \& Estrada, C. (2001). The interplay of subjective essentialism and entitativity in the formation of stereotypes. Personality \& Social Psychology Review, 5, 141-155.

Yzerbyt, V. Y., Rogier, A., \& Fiske, S. T. (1998). Group entitativity and social attribution: On translating situational constraints into stereotypes. Personality \& Social Psychology Bulletin, 24, 1089-1103.

\section{NOTES}

1. To address the possible concern that the probabilities were not distributed normally (they were in intervals of $25 \%$ ), we repeated the $t$ test after taking the arcsine of the square root of each proportion (see Hogg $\&$ Craig, 1995), which produced results $(p s<.001)$ similar to those with the untransformed values.

2 . The one exception was the migrabeaner-angorist pair, with the property of favorite color, for which the coherent category was chosen only $36 \%$ of the time (the mean percentage of coherent-category selections increases to $64 \%$ with this problem excluded); we have no compelling explanation for this anomaly.

\section{APPENDIX
Materials Used in Both Experiments (Set A Only)}

The properties used for Problems 1-4 below were, respectively, red to blue, basketball to baseball, Coca Cola to Pepsi, and daffodils to tulips (in Experiment 1); basketball to baseball, red to blue, daffodils to tulips, and Coca Cola to Pepsi (in Experiment 2). Coherent categories are in italics. For Set B (not shown), present coherent categories became incoherent and vice versa (e.g., migrabeaner was given incoherent properties, whereas angorist was given coherent ones).

Problem 1

\begin{tabular}{ll}
\hline \multicolumn{1}{c}{ PENDERITES } & \multicolumn{1}{c}{ FRAGGLERS } \\
\hline Compassionate & Aggressive \\
Hold meditative poses in trees & Hunt desert animals \\
Spend time in kitchens & Spend time in arid climates \\
Wear magnifying glasses & Wear binoculars \\
Carry mathematicians'ph. numbers & Carry bows and arrows \\
\hline
\end{tabular}

\begin{tabular}{ll}
\multicolumn{2}{c}{ Problem 3} \\
\hline \multicolumn{1}{c}{ DAXETICS } & \multicolumn{1}{c}{ FLORITUMITES } \\
\hline Artistic & Precise \\
Melt glass into jewelry & Extract ocean minerals \\
Spend time in their garages & Spend time in offices \\
Wear flameproof clothing & Wear nametags \\
Carry recyclable bottles & Carry blood pressure monitors \\
\hline
\end{tabular}

Problem 2

\begin{tabular}{|c|c|}
\hline$M I G R A B E A N E R S$ & ANGORISTS \\
\hline Industrious & Spiritual \\
\hline Gather crops by machine & Teach cooking therapy \\
\hline Spend time in fields & Spend time in forests \\
\hline Wear earplugs & Wear wetsuits \\
\hline Carry fuel & Carry padded crates \\
\hline \multicolumn{2}{|c|}{ Problem 4} \\
\hline BORINISTS & ZIFFERS \\
\hline Vigorous & Daring \\
\hline Check articles for stat. errors & Play ice Frisbee \\
\hline Spend time in caves & Spend time on frozen lakes \\
\hline Wear athletic shorts & Wear long underwear \\
\hline Carry piano music & Carry whistles \\
\hline
\end{tabular}

